\title{
The existence of positive solution for singular Kirchhoff equation with two parameters
}

Ke $\mathrm{Di}^{1}$ and Baoqiang Yan ${ }^{1 *}$

\section{Abstract}

In this paper, we consider the singular Kirchhoff equation with two parameters

$$
\left\{\begin{array}{l}
-a\left(\int_{\Omega}|\nabla u(x)|^{2} d x\right) \Delta u(x)+K(x) g(u)=\lambda f(x, u)+\mu h(x) \text { in } \Omega, \\
u>0 \text { in } \Omega, \\
u=0 \text { on } \partial \Omega .
\end{array}\right.
$$

By using the sub-supersolution method together with the comparison principle for elliptic equations, we obtain several existence and nonexistence theorems. Our works improve the results in the previous literature.

Keywords: Elliptic problems of Kirchhoff type; Positive solution; Subsolution; Supersolution

\section{Introduction}

In this paper, we study the existence and nonexistence of solutions to the following singular nonlocal elliptic problem with two parameters:

$$
\left\{\begin{array}{l}
-a\left(\int_{\Omega}|\nabla u(x)|^{2} d x\right) \triangle u(x)+K(x) g(u)=\lambda f(x, u)+\mu h(x) \quad \text { in } \Omega, \\
u>0 \text { in } \Omega, \\
u=0 \text { on } \partial \Omega
\end{array}\right.
$$

where $\Omega$ is a smooth bounded domain in $\mathbb{R}^{N}(N \geq 2), a:[0,+\infty) \rightarrow(0,+\infty)$ is a continuous and nondecreasing function with

$$
\inf _{t \in[0,+\infty)} a(t)=a(0)=a_{0}>0
$$

$K, h \in C^{0, \gamma}(\bar{\Omega})$ with $K>0$ and $h>0$ on $\Omega$, and $\lambda$ and $\mu$ are positive real numbers.

This problem is related to the general form of the stationary counterpart of the hyperbolic Kirchhoff equation

$$
\rho \frac{\partial^{2} u}{\partial t^{2}}-\left(\frac{P_{0}}{h}+\frac{E}{2 L} \int_{0}^{L}\left|\frac{\partial u}{\partial x}\right|^{2} d x\right) \frac{\partial^{2} u}{\partial t^{2}}=g(x, u)
$$

\section{空 Springer}

(c) The Author(s) 2019. This article is distributed under the terms of the Creative Commons Attribution 4.0 International License (http://creativecommons.org/licenses/by/4.0/), which permits unrestricted use, distribution, and reproduction in any medium, provided you give appropriate credit to the original author(s) and the source, provide a link to the Creative Commons license, and indicate if changes were made. 
for free vibrations of elastic strings [7]. Kirchhoff's model takes into account the changes in length of the string produced by transverse vibrations, in which $L$ is the length of the string, $\rho$ is the mass density, and $P_{0}$ is the initial tension. Many researchers began an extensive research in equations of this type after Lions [10] put forward a basic framework. For example, Perera and Zhang [17, 30] considered the following Kirchhof-type problems with 4-sublinear case, asymptotically 4-linear case, and 4-superlinear case terms:

$$
\left\{\begin{array}{l}
-\left(a+b \int_{\Omega}|\nabla u(x)|^{2} d x\right) \Delta u(x)=f(x, u) \quad \text { in } \Omega, \\
u=0 \quad \text { on } \partial \Omega .
\end{array}\right.
$$

They obtained nontrivial solutions via the Yang index and the invariant sets of descent flow. Sun and Tang [22] obtained the existence and multiplicity of weak solutions of (1.2) by using the mountain pass theorem, the local linking theorem, the fountain theorem, and the symmetric mountain pass lemma in critical point theory. Utilizing the local linking theory, Yang and Zhang [29] obtained the nontrivial solutions of (1.2) with quasilinear terms. Yang and Han [28] obtained infinitely many solutions when $f(x, u)$ is odd by using the fountain theorem. In recent years, many researchers studied the fractional Kirchhoff equation. In [9] and [24] the authors deal with fractional Schrödinger-Kirchhoff equations. In [23] the authors obtained the multiplicity of solutions of a fractional $p$-Laplacian Kirchhoff system. In [16] authors deal with nonlocal fractional problems. In addition, in [25] the authors obtained the local existence and blowup of solutions of nonlocal Kirchhoff diffusion problems. Some other results can be found in $[3-5,11-15,20,26]$, and their references.

Since the method of sub-supersolutions is an important tool to solve the existence of solutions for boundary value problems, naturally, some authors hope to use the theorem of sub-supersolutions to discuss the elliptic problems of Kirchhoff type. However, the nonlocal term brings some difficulties. To overcome the difficulty from the nonlocal term, Alves and Corrêa [1] considered following problem:

$$
\left\{\begin{array}{l}
-a\left(\int_{\Omega}|\nabla u(x)|^{2} d x\right) \Delta u(x)=f(x, u) \quad \text { in } \Omega \\
u=0 \text { on } \partial \Omega
\end{array}\right.
$$

and obtained the existence of positive solutions by using the sub-supersolution method under the condition that $f: \Omega \times \mathbb{R} \rightarrow \mathbb{R}$ is an increasing function. Moreover, Alves and Corrêa [2] dealed with the quasilinear stationary Kirchhoff equation

$$
\left\{\begin{array}{l}
-a\left(\int_{\Omega}|\nabla u(x)|^{2} d x\right) \Delta u(x)=f(x, u, \nabla u) \quad \text { in } \Omega, \\
u=0 \text { on } \partial \Omega
\end{array}\right.
$$

and using the pseudomonotone operator theory, they established the existence of weak solutions by constructing a supersolution and a family of subsolutions. Recently, Yan, O’Regan, and Agarwal [27] discussed the following problem:

$$
\left\{\begin{array}{l}
-a\left(\int_{\Omega}|\nabla u(x)|^{2} d x\right) \Delta u(x)=F(x, u) \quad \text { in } \Omega \\
u>0 \text { in } \Omega \\
u=0 \text { on } \partial \Omega
\end{array}\right.
$$


They presented some new definitions of a sub-supersolution to problem (1.5) and obtained the existence of classical solution to problem (1.5) when $F(x, u)=\lambda u^{q}-u^{p+1}(0<q<1$, $p>0)$.

Motivated by the ideas introduced in [6], we suppose that $f: \bar{\Omega} \times[0, \infty) \rightarrow[0, \infty)$ is a Hölder-continuous function that is positive on $\bar{\Omega} \times(0, \infty)$. We also assume that $f$ is nondecreasing with respect to the second variable and is sublinear, that is,

(f1) the mapping $(0, \infty) \ni s \mapsto \frac{f(x, s)}{s}$ is nonincreasing for all $x \in \bar{\Omega}$;

(f2) $\lim _{s \rightarrow 0} \frac{f(x, s)}{s}=+\infty$ and $\lim _{s \rightarrow+\infty} \frac{f(x, s)}{s}=0$ uniformly in $x \in \bar{\Omega}$;

(f3) there exists $n_{0}>0$ such that $\lambda^{n_{0}} f(x, s) \geq f\left(x, \lambda^{n_{0}+1} s\right)$ for all $\lambda \geq 1$.

We assume that $g \in C^{0, \gamma}(0, \infty)$ is a nonnegative and nonincreasing function satisfying:

(g1) $\lim _{s \rightarrow 0} g(s)=+\infty$;

(g2) there exist $C, \delta_{0}>0$ and $\tau \in(0,1)$ such that $g(s) \leq C s^{-\tau}$ for all $s \in\left(0, \delta_{0}\right)$.

Example The function $f(s)=s^{\alpha}(0<\alpha<1)$ fulfills (f1)-(f3), whereas $g(s)=\left(s^{\alpha}+s^{\beta}\right)^{-1}(0<$ $\alpha<1, \beta>1$ ) satisfies assumptions (g1)-(g2).

Denote $D=\left\{u \in C^{2}(\Omega) \cap C(\bar{\Omega}) ; g(u) \in L^{1}(\Omega)\right\}$.

We show in this paper that (1.1) has at least one solution in $D$ for $\lambda$ belonging to a certain range and any $\mu>0$. We also prove that in some cases, (1.1) has no solutions in $D$, provided that $\lambda$ and $\mu$ are sufficiently small.

\section{Remark 1.1}

(i) If $u \in D, v \in C^{2}(\Omega) \cap C(\bar{\Omega})$, and $0<u<v$ in $\Omega$, then $v \in D$.

(ii) Let $u \in C^{2}(\Omega) \cap C(\bar{\Omega})$ be a solution of (1.1). Then $u \in D$ if and only if $\Delta u \in L^{1}(\Omega)$.

Our main results are the following.

Theorem 1.1 Assume that $f$ satisfies (f1)-(f3) and g satisfies (g1)-(g2).

(1) If $\lim _{\lambda \rightarrow+\infty} \frac{\lambda}{a\left(\lambda^{2+2 n}\right)}=+\infty$, then there exists $\bar{\lambda}$ such that (1.1) has at least one solution in $D$ for all $\lambda>\bar{\lambda}$ and $\mu>0$.

(2) There exist $\lambda^{*}$ and $\mu_{*}$ small enough such that (1.1) has no solution in $D$ for all $\lambda<\lambda^{*}$ and $\mu<\mu_{*}$.

Theorem 1.2 Assume that $f$ satisfies (f1)-(f2). If $\int_{0}^{1} g(s) d s=+\infty$, then (1.1) has no solution in $D$ for any $\lambda, \mu>0$.

This paper is organized as follows. Some preliminary lemmas are given in Sect. 2, and Sect. 3 is devoted to proofs of the results. Some ideas also come from [19] and [18].

\section{Preliminaries}

In this section, we consider the general problem

$$
\left\{\begin{array}{l}
-a\left(\int_{\Omega}|\nabla u(x)|^{2} d x\right) \triangle u(x)=F_{1}(x, u)+F_{2}(x, u) \text { in } \Omega, \\
u=0 \text { on } \partial \Omega .
\end{array}\right.
$$

Let $F(x, u):=F_{1}(x, u)+F_{2}(x, u)$. 
Definition 2.1 The pair of functions $\alpha, \beta \in C^{1}(\bar{\Omega}) \cap C^{2}(\Omega)$ are a subsolution and supersolution of (2.1) if $\alpha(x) \leq \beta(x)$ for $x \in \Omega$ and

$$
\begin{aligned}
& \left\{\begin{array}{l}
-\triangle \alpha(x) \leq \frac{1}{b_{0}} F_{1}(x, \alpha(x))+\frac{1}{a_{0}} F_{2}(x, \alpha(x)), \quad x \in \Omega, \\
\left.\alpha\right|_{\partial \Omega} \leq 0,
\end{array}\right. \\
& \left\{\begin{array}{l}
-\triangle \beta(x) \geq \frac{1}{a_{0}} F_{1}(x, \beta(x))+\frac{1}{b_{0}} F_{2}(x, \beta(x)), \quad x \in \Omega, \\
\left.\beta\right|_{\partial \Omega} \geq 0,
\end{array}\right.
\end{aligned}
$$

where $a_{0}=a(0)$ and $b_{0}=a\left(\int_{\Omega} H(x)^{2} d x\right), E \in L^{p}(\Omega)(p>N)$; here

$$
\begin{aligned}
& E(x)=\sup _{u \in[\alpha(x), \beta(x)]}|F(x, u)|, \quad x \in \bar{\Omega}, \\
& H(x)=\frac{1}{a_{0}} \int_{\Omega}\left|G_{x}(x, y)\right| E(y) d y, \quad x \in \bar{\Omega},
\end{aligned}
$$

$G(x, y)$ is the Green function for $-\Delta u(x)=h$, and $\left.u\right|_{\partial \Omega}=0$.

Lemma 2.2 (see [27]) Let $\Omega \subseteq \mathbb{R}^{N}(N \geq 1)$ be a smooth bounded domain. Let $F: \Omega \times \mathbb{R} \rightarrow$ $\mathbb{R}$ be a continuous function. Let $\alpha$ and $\beta$ be the subsolution and supersolution of (2.1), respectively. If

$$
F_{1}(x, u) \geq 0, \quad F_{2}(x, u) \leq 0, \quad \forall x \in \Omega, \alpha(x) \leq u \leq \beta(x)
$$

then problem (2.1) has at least one solution $u$ such that

$$
\alpha(x) \leq u(x) \leq \beta(x) \quad \text { for all } x \in \bar{\Omega} .
$$

Let $\varphi_{1}$ be the normalized positive eigenfunction corresponding to the first eigenvalue $\lambda_{1}$ of the problem

$$
\left\{\begin{array}{l}
-\triangle u(x)=\lambda u \quad \text { in } \Omega, \\
u=0 \quad \text { on } \partial \Omega .
\end{array}\right.
$$

Lemma 2.3 (see [8]) $\int_{\Omega} \varphi_{1}^{-s}<\infty$ if and only if $s<1$

Lemma 2.4 (see [21]) Let $F: \bar{\Omega} \times(0, \infty) \rightarrow \mathbb{R}$ be a Hölder-continuous function with exponent $\gamma \in(0,1)$ on each compact subset of $\bar{\Omega} \times(0, \infty)$ satisfying:

(F1) $\lim _{s \rightarrow \infty} \sup \left(s^{-1} \max _{x \in \bar{\Omega}} F(x, s)\right)<\lambda_{1}$;

(F2) for each $t>0$, there exists a constant $D(t)>0$ such that

$$
F(x, r)-F(x, s) \geq-D(t)(r-s) \quad \text { for } x \in \bar{\Omega} \text { and } r \geq s \geq t
$$

(F3) there exist $\eta_{0}>0$ and an open subset $\Omega_{0} \subset \Omega$ such that

$$
\min _{x \in \bar{\Omega}} F(x, s) \geq 0 \quad \text { for } x \in\left(0, \eta_{0}\right)
$$


and

$$
\lim _{s \rightarrow 0} \frac{F(x, s)}{s}=+\infty \quad \text { uniformly in } x \in \Omega_{0}
$$

Then for any nonnegative function $\varphi_{0} \in C^{2, \gamma}(\partial \Omega)$, the problem

$$
\left\{\begin{array}{l}
-\Delta u(x)=F(x, u) \quad \text { in } \Omega \\
u>0 \quad \text { in } \Omega \\
u=\varphi_{0} \quad \text { on } \partial \Omega
\end{array}\right.
$$

has at least one positive solution $u \in C^{2, \gamma}(G) \cap C(\bar{\Omega})$ for any compact set $G \subset \Omega \cup\{x \in$ $\left.\partial \Omega ; \varphi_{0}(x)>0\right\}$.

Lemma 2.5 (see [21]) Let $F: \bar{\Omega} \times(0, \infty) \rightarrow \mathbb{R}$ be a continuous function such that the mapping $(0, \infty) \ni s \mapsto \frac{F(x, s)}{s}$ is strictly decreasing at each $x \in \Omega$. Assume that there exists $\nu, \omega \in C^{2}(\Omega) \cap C(\bar{\Omega})$ such that:

(a) $\triangle \omega+F(x, \omega) \leq 0 \leq \Delta v+F(x, v)$ in $\Omega$;

(b) $v, \omega>0$ in $\Omega$ and $v \leq \omega$ on $\partial \Omega$;

(c) $\triangle v \in L^{1}(\Omega)$.

Then $v \leq \omega$ in $\Omega$.

We observe that the hypotheses of Lemmas 2.4 and 2.5 are fulfilled for

$$
\Phi_{\lambda}(x, s)=\frac{1}{a_{0}}(\lambda f(x, s)+h(x)) .
$$

Lemma 2.6 Letf satisfy(f1)-(f2). Then for any $\lambda, \mu>0$, according to Lemmas 2.4 and 2.5, the boundary value problem

$$
\left\{\begin{array}{l}
-\Delta U(x)=\lambda f(x, U)+\mu h(x) \quad \text { in } \Omega \\
U>0 \text { in } \Omega \\
U=0 \text { on } \partial \Omega
\end{array}\right.
$$

has a unique solution $U_{\lambda, \mu} \in C^{2, \gamma}(\Omega) \cap C(\bar{\Omega})$.

\section{Proofs of main theorems}

Denote $K^{*}=\max _{x \in \bar{\Omega}} K(x), K_{*}=\min _{x \in \bar{\Omega}} K(x)$.

Proof of Theorem 1.1 According to Lemma 2.6, the boundary value problem

$$
\left\{\begin{array}{l}
-\Delta U(x)=\frac{1}{a_{0}}(f(x, U)+h(x)) \quad \text { in } \Omega \\
U>0 \text { in } \Omega \\
U=0 \text { on } \partial \Omega
\end{array}\right.
$$


has a unique solution $U \in C^{2, \gamma}(\Omega) \cap C(\bar{\Omega})$. Let $H:[0, \infty) \rightarrow[0, \infty)$ be such that

$$
\left\{\begin{array}{l}
H^{\prime \prime}(t)=g(H(t)) \quad \text { for all } t>0 \\
H^{\prime}(0)=H(0)=0
\end{array}\right.
$$

Obviously, $H \in C^{2}(0,+\infty) \cap C^{1}[0,+\infty)$ exists by our assumption (g2). From (3.2) it follows that $H^{\prime \prime}$ is nonincreasing, whereas $H$ and $H^{\prime}$ are nondecreasing on $(0, \infty)$. Using this fact and applying the mean value theorem, we deduce that, for all $t>0$, there exist $\xi_{t}^{1}, \xi_{t}^{2} \in(0, t)$ such that

$$
\frac{H(t)}{t}=\frac{H(t)-H(0)}{t-0}=H^{\prime}\left(\xi_{t}^{1}\right) \leq H^{\prime}(t)
$$

and

$$
\frac{H^{\prime}(t)}{t}=\frac{H^{\prime}(t)-H^{\prime}(0)}{t-0}=H^{\prime}\left(\xi_{t}^{2}\right) \geq H^{\prime \prime}(t) .
$$

These inequalities imply

$$
H(t) \leq t H^{\prime}(t) \leq 2 H(t) \text { for all } t>0
$$

Hence

$$
1 \leq \frac{t H^{\prime}(t)}{H(t)} \leq 2 \text { for all } t>0
$$

On the other hand, by (g2) and (3.2) there exists $\eta>0$ such that

$$
\left\{\begin{array}{l}
H(t) \leq \delta_{0} \quad \text { for all } t \in(0, \eta) \\
H^{\prime \prime}(t) \leq C H^{-\tau}(t) \quad \text { for all } t \in(0, \eta)
\end{array}\right.
$$

which yields

$$
H(t) \leq c t^{\frac{2}{1+\tau}} \quad \text { for all } t \in(0, \eta)
$$

where $c>0$ is a constant.

Set

$$
\alpha_{\lambda, \mu}=M H\left(\varphi_{1}\right)
$$

where $M$ is a positive constant. Then we can get

$$
\begin{aligned}
- & \Delta \alpha_{\lambda, \mu}+\frac{1}{a_{0}} K(x) g\left(\alpha_{\lambda, \mu}\right) \\
& =\lambda_{1} M H^{\prime}\left(\varphi_{1}\right) \varphi_{1}+\frac{1}{a_{0}} K(x) g\left(M H\left(\varphi_{1}\right)\right)-M g\left(H\left(\varphi_{1}\right)\right)\left|\nabla \varphi_{1}\right|^{2} \quad \text { in } \Omega .
\end{aligned}
$$


Fix $M \geq 1$. The monotonicity of $g$ leads to

$$
g\left(M H\left(\varphi_{1}\right)\right) \leq g\left(H\left(\varphi_{1}\right)\right) \text { in } \Omega,
$$

and by (3.6)

$$
\begin{aligned}
- & \Delta \alpha_{\lambda, \mu}+\frac{1}{a_{0}} K(x) g\left(\alpha_{\lambda, \mu}\right) \\
& \leq \lambda_{1} M H^{\prime}\left(\varphi_{1}\right) \varphi_{1}+\frac{1}{a_{0}} K^{*} g\left(H\left(\varphi_{1}\right)\right)-M g\left(H\left(\varphi_{1}\right)\right)\left|\nabla \varphi_{1}\right|^{2} \text { in } \Omega .
\end{aligned}
$$

By Hopf's maximum principle there exist $\delta_{0}$ and $\Sigma \subset \Omega$ such that

$$
\begin{aligned}
& \left|\nabla \varphi_{1}\right| \geq \delta_{0} \quad \text { in } \Omega \backslash \Sigma, \\
& \left|\varphi_{1}\right| \geq \delta_{0} \quad \text { in } \Sigma .
\end{aligned}
$$

On $\Omega \backslash \Sigma$, we choose $M \geq M_{1}=\max \left\{1, \frac{K^{*}}{a_{0} \delta^{2}}\right\}$. Then we have

$$
\frac{1}{a_{0}} K^{*} g\left(H\left(\varphi_{1}\right)\right) \leq M g\left(H\left(\varphi_{1}\right)\right)\left|\nabla \varphi_{1}\right|^{2} \quad \text { in } \Omega \backslash \Sigma \text {. }
$$

Choosing $M \geq \max \left\{M_{1}, \frac{K^{*} g\left(H\left(\delta_{0}\right)\right)}{a_{0} \lambda_{1} H^{\prime}\left(\delta_{0}\right) \delta_{0}}\right\}$, we have

$$
\frac{1}{a_{0}} K^{*} g\left(H\left(\varphi_{1}\right)\right) \leq \lambda_{1} M H^{\prime}\left(\varphi_{1}\right) \varphi_{1} \quad \text { in } \Sigma
$$

It follows from (3.7)-(3.9) that

$$
-\triangle \alpha_{\lambda, \mu}+\frac{1}{a_{0}} K(x) g\left(\alpha_{\lambda, \mu}\right) \leq 2 \lambda_{1} M H^{\prime}\left(\varphi_{1}\right) \varphi_{1} \quad \text { in } \Omega
$$

Since $\varphi_{1}>0$ in $\Omega$, from (3.3) we have

$$
1 \leq \frac{H^{\prime}\left(\varphi_{1}\right) \varphi_{1}}{H\left(\varphi_{1}\right)} \leq 2 \quad \text { in } \Omega
$$

Then (3.10) and (3.11) yield

$$
-\triangle \alpha_{\lambda, \mu}+\frac{1}{a_{0}} K(x) g\left(\alpha_{\lambda, \mu}\right) \leq 4 \lambda_{1} M H\left(\varphi_{1}\right)=4 \lambda_{1} \alpha_{\lambda, \mu} \quad \text { in } \Omega .
$$

Take $A_{0}=4 a_{0} \lambda_{1} c^{-1}|\alpha(x)|_{\infty}$, where $c=\inf _{x \in \bar{\Omega}} f\left(x,\left|\alpha_{\lambda, \mu}\right|_{\infty}\right)>0$. If $\lambda>A_{0}$, then assumption (f1) produces

$$
\frac{\lambda f\left(x, \alpha_{\lambda, \mu}\right)}{a_{0} \alpha_{\lambda, \mu}} \geq \frac{A_{0} f\left(x,\left|\alpha_{\lambda, \mu}\right|_{\infty}\right)}{a_{0}|\alpha(x)|_{\infty}} \geq 4 \lambda_{1} \quad \text { for all } x \in \Omega .
$$

Combined with (3.12), this gives

$$
-\triangle \alpha_{\lambda, \mu}+\frac{1}{a_{0}} K(x) g\left(\alpha_{\lambda, \mu}\right) \leq \frac{1}{a_{0}} \lambda f\left(x, \alpha_{\lambda, \mu}\right) \quad \text { in } \Omega .
$$


Denote $\Omega_{0}=\left\{x \in \Omega ; \varphi_{1}(x)<\eta\right\}$. By (3.4) and (3.5) it follows that

$$
\begin{aligned}
& g\left(\alpha_{\lambda, \mu}\right)=g\left(M H\left(\varphi_{1}\right)\right) \leq g\left(H\left(\varphi_{1}\right)\right) \leq C H^{-\tau}\left(\varphi_{1}\right) \leq C_{0} \varphi_{1}^{\frac{-2 \tau}{1+\tau}} \quad \text { in } \Omega_{0}, \\
& g\left(\alpha_{\lambda, \mu}\right) \leq g(M H(\eta)) \quad \text { in } \Omega \backslash \Omega_{0} .
\end{aligned}
$$

These estimates combined with Lemma 2.3 yield $g\left(\alpha_{\lambda, \mu}\right) \in L^{1}(\Omega)$, and so $\triangle \alpha_{\lambda, \mu} \in L^{1}(\Omega)$.

Set

$$
\beta_{\lambda, \mu}=\lambda^{n_{0}+1} U
$$

where $\lambda \geq \max \{1, \mu\}$.

We now prove that there exists $A_{0}$ such that $\alpha_{\lambda, \mu} \leq \beta_{\lambda, \mu}$ for all $\lambda>A_{0}$.

Since

$$
\begin{aligned}
\triangle \beta_{\lambda, \mu}+\frac{1}{a_{0}}\left(\lambda f\left(x, \beta_{\lambda, \mu}\right)+h(x)\right) & =\lambda^{n_{0}+1} \Delta U+\frac{1}{a_{0}}\left(\lambda f\left(x, \lambda^{n_{0}+1} U\right)+h(x)\right) \\
& \leq \lambda^{n_{0}+1}\left(\triangle U+\frac{1}{a_{0}}(f(x, U)+h(x))\right)=0,
\end{aligned}
$$

we can get

$$
\begin{aligned}
& \triangle \beta_{\lambda, \mu}+\Phi_{\lambda}\left(x, \beta_{\lambda, \mu}\right) \leq 0 \leq \Delta \alpha_{\lambda, \mu}+\Phi_{\lambda}\left(x, \alpha_{\lambda, \mu}\right) \quad \text { in } \Omega, \\
& \alpha_{\lambda, \mu}, \beta_{\lambda, \mu}>0 \quad \text { in } \Omega, \\
& \alpha_{\lambda, \mu}=\beta_{\lambda, \mu} \quad \text { on } \partial \Omega, \\
& \triangle \alpha_{\lambda, \mu} \in L^{1}(\Omega) .
\end{aligned}
$$

By Lemma 2.5 it follows that $\alpha_{\lambda, \mu} \leq \beta_{\lambda, \mu}$ on $\bar{\Omega}$ for all $\lambda>A_{0}$.

Define

$$
\begin{aligned}
& E_{1}(x)=\lambda f\left(x, \beta_{\lambda, \mu}\right)+\mu h(x)+K(x) g\left(\alpha_{\lambda, \mu}\right), \quad x \in \bar{\Omega}, \\
& H_{1}(x)=\frac{1}{a_{0}} \int_{\Omega}\left|G_{x}(x, y)\right| E_{1}(y) d y, \quad x \in \bar{\Omega}, \\
& b_{1}=a\left(\int_{\Omega} H_{1}(x)^{2} d x\right),
\end{aligned}
$$

that is,

$$
\begin{aligned}
& E(x)=\sup _{u \in\left[\alpha_{\lambda, \mu}(x), \beta_{\lambda, \mu}(x)\right]}|F(x, u)| \leq E_{1}(x), \quad x \in \bar{\Omega}, \\
& H(x)=\frac{1}{a_{0}} \int_{\Omega}\left|G_{x}(x, y)\right| E(y) d y \leq H_{1}(x), \quad x \in \bar{\Omega}, \\
& b_{0}=a\left(\int_{\Omega} H(x)^{2} d x\right) \leq b_{1}=a\left(\int_{\Omega} H_{1}(x)^{2} d x\right) .
\end{aligned}
$$


We have

$$
\begin{aligned}
b_{1} & =a\left(\int_{\Omega} H_{1}(x)^{2} d x\right) \\
& =a\left(\int_{\Omega}\left[\frac{1}{a_{0}} \int_{\Omega}\left|G_{x}(x, y)\right|\left(\lambda f\left(y, \lambda^{n_{0}+1} U\right)+\mu h(y)+K(y) g\left(M H\left(\varphi_{1}\right)\right)\right) d y\right]^{2} d x\right) \\
& \leq a\left(\int_{\Omega}\left[\frac{1}{a_{0}} \int_{\Omega}\left|G_{x}(x, y)\right|\left(\lambda f\left(y, \lambda^{n_{0}+1} U\right)+\lambda h(y)+K^{*} g\left(H\left(\varphi_{1}\right)\right)\right) d y\right]^{2} d x\right) \\
& \leq a\left(\left(\frac{\lambda}{a_{0}}\right)^{2} \int_{\Omega}\left[\int_{\Omega}\left|G_{x}(x, y)\right|\left(f\left(y, \lambda^{n_{0}+1} U\right)+h(y)+K^{*} g\left(H\left(\varphi_{1}\right)\right)\right) d y\right]^{2} d x\right) \\
& \leq a\left(\frac{\lambda^{2+2 n_{0}}}{a_{0}^{2}} \int_{\Omega}\left[\int_{\Omega}\left|G_{x}(x, y)\right|\left(f(y, U)+h(y)+K^{*} g\left(H\left(\varphi_{1}\right)\right)\right) d y\right]^{2} d x\right) .
\end{aligned}
$$

Let

$$
C=\left(\frac{1}{a_{0}}\right)^{2} \int_{\Omega}\left[\int_{\Omega}\left|G_{x}(x, y)\right|\left(f(y, U)+h(y)+K^{*} g\left(H\left(\varphi_{1}\right)\right)\right) d y\right]^{2} d x
$$

that is,

$$
b_{1} \leq a\left(C \lambda^{2+2 n_{0}}\right)
$$

where $C$ is a positive constant. Since $\lim _{\lambda \rightarrow+\infty} \frac{\lambda}{a\left(\lambda^{\left.2+2 n_{0}\right)}\right.}=+\infty$, there exists $B_{0}$ such that

$$
\frac{\lambda}{a\left(C \lambda^{2+2 n_{0}}\right)} \geq 4 \lambda_{1} c^{-1}|\alpha|_{\infty} \quad \text { for all } \lambda \geq B_{0}
$$

Choosing $\bar{\lambda}=\max \left\{1, \mu, A_{0}, B_{0}\right\}$, we easily to see that

$$
-\triangle \alpha_{\lambda, \mu} \leq \frac{1}{b_{0}}\left(\lambda f\left(x, \alpha_{\lambda, \mu}\right)+\mu h(x)\right)-\frac{1}{a_{0}} K(x) g\left(\alpha_{\lambda, \mu}\right) \quad \text { in } \Omega \text { for all } \lambda>\bar{\lambda} .
$$

Since

$$
-\triangle \lambda^{n_{0}} U=\frac{1}{a_{0}} \lambda^{n_{0}}(f(x, U)+h(x)) \geq \frac{1}{a_{0}}\left(f\left(x, \lambda^{n_{0}+1} U\right)+h(x)\right) \quad \text { in } \Omega,
$$

we get

$$
-\triangle \lambda^{n_{0}+1} U \geq \frac{\lambda}{a_{0}}\left(f\left(x, \lambda^{n_{0}+1} U\right)+h(x)\right) \quad \text { in } \Omega .
$$

Hence

$$
-\triangle \beta_{\lambda, \mu} \geq \frac{1}{a_{0}}\left(\lambda f\left(x, \beta_{\lambda, \mu}\right)+\mu h(x)\right)-\frac{1}{b_{0}} K(x) g\left(\beta_{\lambda, \mu}\right) \quad \text { in } \Omega \text { for all } \lambda>\bar{\lambda} .
$$

By Lemma 2.2 (1.1) has at least one solution $u_{\lambda, \mu}$ such that $\alpha_{\lambda, \mu} \leq u_{\lambda, \mu} \leq \beta_{\lambda, \mu}$ in $\bar{\Omega}$. Since $g\left(\alpha_{\lambda, \mu}\right) \in L^{1}(\Omega)$, we have $\alpha_{\lambda, \mu} \in D$. By Remark 1.1 we deduce that $u_{\lambda, \mu} \in D$. Hence, for all $\lambda \geq \bar{\lambda}$ and $\mu>0$, problem (1.1) has at least one solution $u_{\lambda, \mu} \in D$. 
Nonexistence for $\lambda, \mu$ small. Let $\lambda, \mu>0$. Set

$$
\Theta_{\lambda, \mu}(x . s)=\frac{1}{a_{0}}(\lambda f(x, s)+\mu h(x)-K(x) g(s)) \text { in } \Omega .
$$

Since $K_{*}>0$, assumption (g1) implies $\lim _{s \rightarrow 0} \Theta_{\lambda, \mu}(x . s)=-\infty$ uniformly in $x \in \bar{\Omega}$. Then there exists $c>0$ such that

$$
\Theta_{\lambda, \mu}(x . s)<0 \quad \text { for all }(x, s) \in \bar{\Omega} \times(0, c) .
$$

Let $s \geq c$. From (f1) we deduce

$$
\frac{\Theta_{\lambda, \mu}(x . s)}{s} \leq \frac{1}{a_{0}}\left(\lambda \frac{f(x, s)}{s}+\mu \frac{h(x)}{s}\right) \leq \frac{1}{a_{0}}\left(\lambda \frac{f(x, c)}{c}+\mu \frac{|h|_{\infty}}{s}\right)
$$

for all $x \in \bar{\Omega}$. Fix $\mu \leq \frac{c \lambda_{1} a_{0}}{2|h|_{\infty}}$ and let $M=\sup _{x \in \bar{\Omega}} \frac{f(x, c)}{a_{0} c}>0$. From the above inequality we have

$$
\frac{\Theta_{\lambda, \mu}(x . s)}{s} \leq \lambda M+\frac{\lambda_{1}}{2} \quad \text { for all }(x, s) \in \bar{\Omega} \times(c,+\infty) .
$$

Set $A(\lambda)=\lambda M$. Inequalities (3.13) and (3.14) yield

$$
\Theta_{\lambda, \mu}(x . s) \leq A(\lambda) s+\frac{\lambda_{1}}{2} s \quad \text { for all }(x, s) \in \bar{\Omega} \times(0,+\infty) .
$$

Moreover, $A(\lambda) \rightarrow 0$ as $\lambda \rightarrow 0$. If (1.1) has a solution $u_{\lambda, u}$, then

$$
\begin{aligned}
\lambda_{1} \int_{\Omega} u_{\lambda, \mu}^{2}(x) d x & \leq \int_{\Omega}\left|\nabla u_{\lambda, \mu}(x)\right|^{2} d x \\
& =-\int_{\Omega} u_{\lambda, \mu}(x) \triangle u_{\lambda, \mu}(x) d x
\end{aligned}
$$

and using (3.15), we get

$$
\lambda_{1} \int_{\Omega} u_{\lambda, \mu}^{2}(x) d x \leq\left[A(\lambda)+\frac{\lambda_{1}}{2}\right] \int_{\Omega} u_{\lambda, \mu}^{2}(x) d x
$$

Since $A(\lambda) \rightarrow 0$ as $\lambda \rightarrow 0$, this relation leads to a contradiction for $\lambda, \mu>0$ sufficiently small. The proof of Theorem 1.1 is now complete.

Proof of Theorem 1.2 Suppose to the contrary that exist $\lambda$ and $\mu$ such that (1.1) has a solution $u_{\lambda, \mu} \in D$, and by Lemma 2.6 the boundary value problem

$$
\left\{\begin{array}{l}
-\triangle V(x)=\frac{1}{a\left(\int_{\Omega}\left|\nabla u_{\lambda, \mu}\right|^{2} d x\right)}(\lambda f(x, V)+\mu h(x)) \quad \text { in } \Omega \\
V>0 \quad \text { in } \Omega \\
V=0 \quad \text { on } \partial \Omega
\end{array}\right.
$$

has a unique solution $V_{\lambda, \mu} \in C^{2, \gamma}(\Omega) \cap C(\bar{\Omega})$. 
Set

$$
\Psi_{\lambda, \mu}(x, s)=\frac{1}{a\left(\int_{\Omega}\left|\nabla u_{\lambda, \mu}\right|^{2} d x\right)}(\lambda f(x, s)+\mu h(x)) .
$$

Since

$$
\Delta V_{\lambda, \mu}+\Psi_{\lambda, \mu}\left(x, V_{\lambda, \mu}\right) \leq 0 \leq \Delta u_{\lambda, \mu}+\Psi_{\lambda, \mu}\left(x, u_{\lambda, \mu}\right) \quad \text { in } \Omega,
$$

by Lemma 2.5 we get $u_{\lambda, \mu} \leq V_{\lambda, \mu}$ in $\Omega$.

Consider the perturbed problem

$$
\left\{\begin{array}{l}
-\Delta u+K_{*} g(u+\epsilon)=\frac{1}{a\left(\int_{\Omega}\left|\nabla u_{\lambda, \mu}\right|^{2} d x\right)}(\lambda f(x, u)+\mu h(x)) \text { in } \Omega, \\
u>0 \text { in } \Omega, \\
u=0 \quad \text { on } \partial \Omega .
\end{array}\right.
$$

Since $K_{*}>0$, it follows that $u_{\lambda, \mu}$ and $V_{\lambda, \mu}$ are subsolution and supersolution for (3.17), respectively. Then there exists a solution $u_{\epsilon} \in C^{2, \gamma}(\bar{\Omega})$ of (3.17) such that

$$
u_{\lambda, \mu} \leq u_{\epsilon} \leq V_{\lambda, \mu}
$$

Integrating in (3.17), we deduce

$$
-\int_{\Omega} \Delta u_{\epsilon} d x+K_{*} \int_{\Omega} g\left(u_{\epsilon}+\epsilon\right) d x=\frac{1}{a\left(\int_{\Omega}\left|\nabla u_{\lambda, \mu}\right|^{2} d x\right)} \int_{\Omega}\left[\lambda f\left(x, u_{\epsilon}\right)+\mu h(x)\right] d x .
$$

Hence

$$
-\int_{\partial \Omega} \frac{\partial u_{\epsilon}}{\partial n}+K_{*} \int_{\Omega} g\left(u_{\epsilon}+\epsilon\right) d x \leq M
$$

where $M>0$ is a constant. Since $\frac{\partial u_{\epsilon}}{\partial n} \leq 0$ on $\partial \Omega$, relation (3.18) yields $K_{*} \int_{\Omega} g\left(u_{\epsilon}+\epsilon\right) d x \leq$ $M$, and so $K_{*} \int_{\Omega} g\left(V_{\lambda, \mu}+\epsilon\right) d x \leq M$. Thus, for any compact subset $\omega \subset \Omega$, we have

$$
K_{*} \int_{\omega} g\left(V_{\lambda, \mu}+\epsilon\right) d x \leq M
$$

Letting $\epsilon \rightarrow 0$, this relation leads to $K_{*} \int_{\omega} g\left(V_{\lambda, \mu}\right) d x \leq M$. Therefore

$$
K_{*} \int_{\Omega} g\left(V_{\lambda, \mu}\right) d x \leq M
$$

Choose $\delta>0$ sufficiently small and define $\Omega_{\delta}:=\{x \in \Omega$; $\operatorname{dist}(x, \partial \Omega) \leq \delta\}$. Taking into account the regularity of the domain, we get that there exists $k>0$ such that

$$
V_{\lambda, \mu} \leq k \operatorname{dist}(x, \partial \Omega) \quad \text { for all } x \in \Omega_{\delta} .
$$

Then

$$
\int_{\Omega} g\left(V_{\lambda, \mu}\right) d x \geq \int_{\Omega_{\delta}} g\left(V_{\lambda, \mu}\right) d x \geq \int_{\Omega_{\delta}} g(k \operatorname{dist}(x, \partial \Omega)) d x=+\infty,
$$


which contradicts (3.19). It follows that problem (1.1) has no solution in $D$, and the proof of Theorem 1.2 is now complete.

\author{
Acknowledgements \\ The authors thank the referees for their comments. \\ Funding \\ This work is supported by the National Natural Science Foundation of China (61603226) and the Fund of Natural Science \\ of Shandong Province (ZR2018MA022).
}

\title{
Availability of data and materials
}

Not applicable.

\section{Competing interests}

The authors declare that they have no competing interests.

\section{Authors' contributions}

Both authors contributed equally to the writing of this paper. Both authors read and approved the final manuscript.

\section{Authors' information}

Not applicable.

\section{Publisher's Note}

Springer Nature remains neutral with regard to jurisdictional claims in published maps and institutional affiliations.

Received: 6 November 2018 Accepted: 11 February 2019 Published online: 21 February 2019

\section{References}

1. Alves, C.O., Corrêa, F.J.S.A.: On existence of solutions for a class of problem involving a nonlinear operator. Commun Appl. Nonlinear Anal. 8, 43-56 (2014)

2. Alves, C.O., Corrêa, F.J.S.A.: A sub-supersolution approach for a quasilinear Kirchhoff equation. J. Math. Phys. 56, 591-608 (2015)

3. Alves, C.O., Corrêa, F.J.S.A., Ma, T.M.: Positive solutions for a quasilinear elliptic equation of Kirchhoff type. Comput. Math. Appl. 49, 85-93 (2005)

4. Bensedik, A., Bouchekif, M.: On an elliptic equation of Kirchhoff type with a potential asymptotically linear at infinity. Math. Comput. Model. 49, 1089-1096 (2009)

5. Cheng, B., Wu, X.: Existence results of positive solutions of Kirchhoff type problems. Nonlinear Anal. 71, 4883-4892 (2009)

6. Ghergu, M., Rădulescu, V.: Sublinear singular elliptic problems with two parameters. J. Differ. Equ. 195, $520-536$ (2003)

7. Kirchhoff, G.: Mechanik. Teubner, Leipzig (1883)

8. Lazer, A.C., McKenna, P.J.: On a singular nonlinear elliptic boundary value problem. Proc. Am. Math. Soc. 111, 721-730 (1991)

9. Liang, S., Repovš, D., Zhang, B.: On the fractional Schrödinger-Kirchhoff equations with electromagnetic fields and critical nonlinearity. Comput. Math. Appl. 75, 1778-1794 (2018)

10. Lions, J.L.: On some questions in boundary value problems of mathematical physics. In: Contemporary Developments in Continuum Mechanics and Partial Differential Equations. North-Holland Mathematics Studies, vol. 36, pp. 284-346 (1977)

11. Lu, S.S.: Multiple solutions for a Kirchhoff-type equation with general nonlinearity. Adv. Nonlinear Anal. 7, 293-306 (2016)

12. Ma, T.: Remarks on an elliptic equation of Kirchhoff type. Nonlinear Anal. 63, 1967-1977 (2005)

13. Mao, A., Luan, S.: Sign-changing of a class nonlocal quasilinear elliptic boundary value problems. J. Math. Anal. Appl. 383, 239-243 (2011)

14. Mao, A., Zhang, Z.: Sign-changing and multiple solutions of Kirchhoff type problems without the P.S. condition. Nonlinear Anal., Theory Methods Appl. 70, 1275-1287 (2009)

15. Mao, A., Zhu, X.: Existence and multiplicity results for Kirchhoff problems. Mediterr. J. Math. 14, 58 (2017). https://doi.org/10.1007/s00009-017-0875-0

16. Molica Bisci, G., Rădulescu, V., Servadei, R.: Variational Methods for Nonlocal Fractional Problems. Encyclopedia of Mathematics and Its Applications, vol. 162. Cambridge University Press, Cambridge (2016)

17. Perera, K., Zhang, Z.: Nontrivial solutions of Kirchhoff-type problems via the Yang index. J. Differ. Equ. 221, 246-255 (2006)

18. Rădulescu, V.: Singular phenomena in nonlinear elliptic problems: from blow-up boundary solutions to equations with singular nonlinearities. In: Handbook of Differential Equations: Stationary Partial Differential Equations, vol. 4, Chap. 7, pp. 485-593 (2007)

19. Rădulescu, V.: Combined effects in nonlinear singular elliptic problems with convection. Rev. Roum. Math. Pures Appl. 53, 543-553 (2008)

20. Ricceri, B.: On an elliptic Kirchhoff-type problem depending on two parameters. J. Glob. Optim. 46, 543-549 (2010)

21. Shi, J., Yao, M.: On a singular nonlinear semilinear elliptic problem. Proc. R. Soc. Edinb. 128, 1389-1401 (1998)

22. Sun, J., Tang, C.: Existence and multiplicity of solutions for Kirchhoff type equations. Nonlinear Anal., Theory Methods Appl. 74, 1212-1222 (2011) 
23. Xiang, M., Rădulescu, V., Zhang, B.: Multiplicity of solutions for a class of quasilinear Kirchhoff system involving the fractional $p$-Laplacian. Nonlinearity 29, 3186-3205 (2016)

24. Xiang, M., Rădulescu, V., Zhang, B.: Combined effects for fractional Schrödinger-Kirchhoff systems with critical nonlinearities. ESAIM Control Optim. Calc. Var. 24, 1249-1273 (2018)

25. Xiang, M., Rădulescu, V., Zhang, B.: Nonlocal Kirchhoff diffusion problems: local existence and blow-up of solutions. Nonlinearity 31, 3228-3250 (2018)

26. $\mathrm{Xu}, \mathrm{L}$., Chen, H.: Ground state solutions for Kirchhoff-type equations with a general nonlinearity in the critical growth. Adv. Nonlinear Anal. 7, 535-546 (2018)

27. Yan, B., O'Regan, D., Agarwal, R.P.: The existence of positive solutions for Kirchhoff-type problems via the sub-supersolution method. An. Ştiinţ. Univ. 'Ovidius' Constanţa 26, 5-41 (2018)

28. Yang, M., Han, Z: Existence and multiplicity results for Kirchhoff type problems with four-superlinear potentials. Appl. Anal. 91, 2045-2055 (2012)

29. Yang, Y., Zhang, J.: Nontrivial solutions of a class of nonlocal problems via local linking theory. Appl. Math. Lett. 23, 377-380 (2010)

30. Zhang, Z., Perera, K.: Sign changing solutions of Kirchhoff type problems via invariant sets of descent flow. J. Math. Anal. Appl. 317, 456-463 (2006)

\section{Submit your manuscript to a SpringerOpen ${ }^{\circ}$ journal and benefit from:}

- Convenient online submission

- Rigorous peer review

- Open access: articles freely available online

- High visibility within the field

Retaining the copyright to your article

Submit your next manuscript at $\boldsymbol{~ s p r i n g e r o p e n . c o m ~}$ 